\title{
LA COMPRENSIÓN DE LECTURA EN TEXTOS DE CIENCIAS NATURALES
}

\author{
SANTELICES CUEVAS, $L$. \\ Pontificia Universidad Católica de Chile.
}

\section{SUMMARY}

There are many linguistic factors and pre-scientific notions related to the process of reading comprehension of written materials used in science teaching.

This paper shows these factors and proposes some strategies for increasing the reading comprehension of texts related to science teaching.

Los materiales escritos, tales como libros, guías de laboratorio y horas de trabajo, juegan un papel preponderante en la enseñanza de las Ciencias Naturales. Sin embargo, constantemente los profesores de ciencias se quejan de que los niños no pueden leer los textos y señalan que en consecuencia se torna, entonces, más difícil aprender ciencias (Santesteban y Koran 1977) (Stuart 1982). Por otra parte, el empleo del lenguaje en la enseñanza de las Ciencias Naturales está siendo objeto de mayor atención en la última década. En efecto, el lenguaje está siendo progresivamente considerado como una variable fundamental en el proceso de adqui. sición de conceptos científicos (Medway 1976, Llorens y col. 1987) y no sólo como un medio de comunicación de esta disciplina.

Sin embargo, esta relación entre procesos cognoscitivos y lenguaje es antigua. A partir de la década de 1960 , ya numerosos autores, admitiendo un origen diferente al pensamiento y el lenguaje, reconocen su interacción (Vygotski 1962, Ausubel 1968, Whorf 1971, Sapir 1970 y Luria 1980). Además, se ha demonstrado que existe correlación significativa entre el grado de madurez lingüústica, medida por el nivel de complejidad sintáctica con que se expresan los alumnos, y su desarrollo cognitivo. (Lawson y Shepherd 1979). También se ha comprobado que la falta de coherencia entre las estructuras cognitivas del niño y el vocabulario empleado en la enseñanza da lugar a problemas en el contexto del aprendizaje de las Ciencias Naturales (Gardner 1972).

Se postula que la estructura y el contenido de los textos de Ciencias Naturales son vías de acceso a la comprensión de los conceptos en esta disciplina. (Castañeda y col. 1987). Se señala además, que las diferencias entre los esquemas o estructuras cognoscitivas del niño y los contenidos que se plantean en los textos de ciencias pueden actuar como barreras críticas para la comprensión (Hawkins 1978). Estudios que comparan el modo de actuar de expertos y de principiantes frente a la tarea de resolver problemas de ciencias demuestran que los primeros son capaces de comprender el problema y describirlo cualitativamente, en tanto los segundos los suelen hacer incorrectamente, no porque tengan dificuitad en manipular, por ejemplo, las relaciones matemáticas, sino a causa de las dificultades conceptuales implicadas en su enunciado (Gamble 1986). 
Los antecedentes anteriores permiten inferir que existe un conjunto de variables lingüísticas relacionado con los procesos de comprensión de los textos utilizados en la enseñanza de las ciencias. Así, en este artículo se analizarán en primer lugar, las variables más relevantes que repercuten en el proceso de comprensión en lectura de materiales escritos de Ciencias Naturales; y en segundo lugar, se propondrán estrategias de acción para mejorar la comprensión de textos de apoyo para la enseñanza de las Ciencias Naturales, que sobre la base de las variables analizadas faciliten al niño la concep. tualización científica.

\section{VARIABLES OUE INCIDEN EN LA COM- PRENSION LECTORA DE LOS TEXTOS DE CIENCIAS NATURALES}

De acuerdo con el modelo interactivo del proceso lector postulado por Rumelhart (1976), la lectura implica considerar simultáneamente la activación y utilización de los esquemas o estructuras cognoscitivas del lector, su motivación intrínseca y el procesamiento de los elementos derivados del texto.

Se postula además que no hay un proceso único de comprensión de los materiales escritos y que las demandas impuestas al lector varían en diferentes situaciones y en función de diferentes formas de lenguaje utilizado en ellos. (Van Dijk y Kintsch 1983). Britton Geym Meyer y Penland (1982) plantean que hay dos grupos de variables que demandan capacidad de procesamiento cognoscitivo al lector: variables asociadas con el contenido del texto (semánticas) y variables de orden estructural (sintaxis y vocabulario); éstas últimas son relativamente independientes del contenido pero específicas a la expresión de éste.

Holley, Dansereau y Fender (1981) proponen por otra parte que la naturaleza de los estímulos puede inducir tanto al tipo de procesamiento cerebral de la información, como a la estrategia predominante para comprender el texto.

Meyer (1984) plantea también que diferentes repre. sentaciones cognitivas se podrían construir a partir de una codificación selectiva de una clase de información sobre otra. Por otra parte, investigaciones realizadas directamente en relación a la comprensión de materiales de ciencias destacan la necesidad de considerar tanto la estructura como los contenidos de los textos, y las estrategias cognitivas que utiliza el alumno para acceder al significado (Castañeda y col. 1987).

Estos mismos investigadores afirman que la comprensión de los textos de ciencias requiere generalmente que el lector posea un conocimiento formal específico en relación a los temas tratados y que además sea capaz de penetrar en el núcleo de información contenido en una afirmación, principio o ley científica. Sobre la base de los antecedentes presentados se hace necesario analizar con mayor profundidad dos variables mencio- nadas con mayor frecuencia en ellos: $a$ ) el conocimiento previo del lector y b) el vocabulario, sintaxis y relaciones tópicas del contenido entregado en el texto.

\section{a) Conocimiento previo del lector}

Durante muchos años se ha utilizado el texto de ciencias como un medio fundamental de apoyo al proceso de enseñanza de los conceptos científicos; sin embargo, no está claro si estos medios han tenido éxito en este aspecto, por cuanto son numerosas las investigaciones que comprueban serias dificultades en el proceso de conceptualización científica (Moreira y Novack 1986, Herron 1979, Gilbert y Osborne 1980).

Desde esta perspectiva y con el fin de precisar la acepción que se da en el contexto de la ciencia al término "concepto", parece conveniente iniciar el análisis de esta problemática con la revisión de las variadas posiciones teóricas frente al problema de su definición. Estas posiciones sin embargo, muestran una constante: "los conceptos serían un conjunto de rasgos abstractos que corresponden a la información común de todos los miembros de la misma clase" (Moreno 1985). Por ejemplo; el concepto "perro" encierra un conjunto de características aplicables a todos los individuos de esa clase: perros, y este conjunto de caracteres o atributos esenciales permite la existencia del concepto perro en la mente de todas las personas de una comunidad.

Gagné y Briggs (1974) distinguen entre concepto concreto y concepto definido. El primero permite identificar una propiedad del objeto, o sea un atributo propio. El segundo requiere la habilidad del individuo para expresar el significado de una clase de objetos o sus relaciones .

La posibilidad de conceptualizar parece estar estrechamente relacionada con el lenguaje, razón por la cual su empleo en la formación de conceptos científicos es objeto de creciente interés. En efecto, el lenguaje es un factor fundamental en el proceso de adquisición de conceptos (Medway 1980, Luria 1980). La utilización de paiabras del lenguaje ordinario dentro del contexto científico, así como las palabras científicas incorporadas al lenguaje ordinario han hecho surgir muchos problemas en relactón al aprendizaje de las ciencias. Numerosas investigaciones realizadas en este campo han permitido describir que el uso incorrecto o descuidado de la terminología induce a la formación de errores en la conceptualización científica. (Herron 1979, Gilbert 1980, Cassels y Johnstone 1983, Llorens, Llopis y Dejaime 1987).

Desde esta perspectiva es importante tomar conciencia de que los niños de siete años que ingresan en la enseñanza básica poseen generalmente una serie de conceptos superficiales e imprecisos que se denominan "concepciones espontáneas" o "pre-conceptos" (Pozo y Carretero 1987) los cuales obstaculizan la comprensión de los conceptos científicos. Estos pre-conceptos deberían ser considerados por los profesores de Ciencias Naturales en el momento de diseñar sus materiales de enseñanza en la educación básica. 
Las diversas concepciones espontáneas que tienen los alumnos con respecto a áreas tan diferentes como biología, física, química, medicina comparten las características en cuanto a su naturaleza, a su origen y a su desarrollo que se describen a continuación (Pozo y Carretero 1987):

1o Surgen de un modo natural en la mente del niño, comoconsecuencia de su interacción cotidiana con el mundo que le rodea. Se podrían considerar como "ciencia intuitiva" y resultan adecuadas para predecir resultados en esas experiencias comunes (Osborne y Freyberg 1985 ).

$2^{\circ}$ Son construcciones personales del alumno, producto de su personal actividad intelectual.

$3^{2}$ Son científicamente incorrectos, porque a menudo el grado de abstracción limitado que posee el escolar de enseñanza básica restringe su observación a los aspectos superficiales.

4\% Suelen ser implícitas, constituyen "teorías en acción" (Driver 1983, 1986) y muchas veces el alumno no tiene conciencia de poseerlas (metacognición) y por eso tampoco las verbaliza. El niño puede predecir correctamente en función de ellas pero es incapaz de explicar el por qué de su predicción (Pozo 1987a).

$5^{\circ}$ Pueden ser incoherentes o contradictorias entre sí, sobre todo cuando el niño es pequeño y no ha sido capaz de verbalizarlas.

$6^{2}$ Son resistentes al cambio, las concepciones espontáneas o preconceptos en un mismo dominio se relacionan entre sí y se organizan en forma de teorías, adoptando Ia forma de estructuras jerárquicas (Chil, Gloser y Rees 1982), con una función explicativa, ya que sirven para predecir y controlar acontecimientos (Otero y Brincones 1987).

Estos antecedentes permiten inferir que aunque el preconcepto o la "teoría intuitiva" sea errónea desde una perspectiva científica, está basada en una experiencia fenomenológica cotidiana y por elloes difícil modificar.

Numerosas investigaciones han puesto de manifiesto que la eliminación de una hipótesis, mediante su negación, es difícil no sólo para el niño sino también para el adulto (Carretero 1987, García Madruga y Carretero 1985, Delval 1975 y 1977). Podría postularse entonces que las personas tienen una tendencia a no descartar una hipótesis cuando piensan que es cierta, aunque existan ejemplos que muestren su falsedad. Esta afirmación podría apoyarse en investigaciones que han demostrado la resistencia que presentan los niños y adolescentes a abandonar las ideas que espontáneamente se han formado acerca de una situación o fenómeno científico (Carretero 1987).

A lo anterior se agrega otro antecedente importante para la enseñanza de la ciencia. Durante años, los profesores que asumieron la concepción piagetana han pensado que si el pensamiento formal actúa con independencia de los contenidos concretos a los que se aplica, proporcionando a los alumnos instancias para estructurar su pensamiento formal, ellos estarian en condiciones de entender cualquier concepto científico. Sin embargo, se ha comprobado que no todos los esquemas formales de pensamiento se adquieren simultáneamente. Se ha demostrado también que en la resolución đe tareas formales no sólo influye la estructura lógica del problema, sino también el contenido al que éste se refiere. Esta influencia del contenido está mediatizada por las concepciones previas que el sujeto tiene con respecto a ese contenido (Pozo 1987).

Si se acepta que el lector procesa el tex to que lee en función de sus esquemas previos (Condemarín), es posible suponer que el texto de ciencias debería necesa* riamente considerar las concepciones espontáneas o pre-conceptos que aparecen con mayor frecuencia frente a un área de contenido o de conceptos determinada, para que constituya un punto de partida para estructurar la concepción científica real.

\section{b) Vocabulario, sintaxis y relaciones lógicas del contenido}

Eisenberg (1977) señala que la estructura del lenguaje afecta la comprensión y la facilidad de lectura en las Ciencias Naturales.

Existe un vocabulario común a las ciencias, y éste se caracteriza por la precisión de sus términos, los que a menudo tienen en este contex to sólo un significado. Algunos trabajos realizados por Cassels y Johnstone (1983) advierten que la comprensión del vocabulario depende del contexto y de su aceptación, por lo cual muchas veces resulta al niño más difícil utilizar la acepción corriente del término y no la necesaria para completar un esquema científico.

Vygotski (1962) señala que el niño pequeño e incluso el joven, frente a cada palabra asocia un alto número de significados que deben irse reduciendo progresivamente hasta lograr mayor precisión. Sería por tanto importante, frente a la tarea de elaborar un texto en la asignatura, conocer el vocabulario "preteórico" y los significados asignados a cada término en los distintos contextos en que aparecen, para que un niño pudiera leer un término científico y comprenderlo en su sentido común (Gilbert 1980).

También es propio de los escritos científicos, utilizar formas verbales en pasiva, oraciones de estructura compleja que a menudo incluyen gran cantidad de anáforas (palabras omitidas que el lector debe inferir), todo lo cual contribuye a dificultar la comprensión.

El lenguaje científico en general utiliza escasa redun* dancia y la información se presenta a menudo con una relación de cadenas causa-efecto que constituyen focos centrales para la comprensión de las redes conceptuales tratadas.

Según Weidler (1984), todos estos planteamientos deberían ayudar al educador a tomar conciencia de las grandes barreras que puede presentar un texto para la 
comprensión y conceptualización científica del niño y del adolescente.

\section{ESTRATEGIAS PARA MEJORAR LA COMPRENSION EN LA LECTURA DE TEXTOS DE CIENCIAS NATURALES}

Cuando los estudiantes tienen algún conocimiento previo de un aspecto científico ese esquema provee una red para asimilar el nuevo conocimiento. (Anderson 1984, Anderson y Pearson 1984). Sin embargo, los alumnos no integran espontáneamente sus antiguos y nuevos conocimientos. Los pre-conceptos o teorías intuitivas necesitan ser abordadas, Ios nuevos conocimientos deben ser presentados de modo cercano y comprensible y para todo ello el texto y el profesor deben proveer puentes desde los cuales los alumnos accedan al conocimiento científico.

Una forma de construir estos puentes es a través del uso de analogías. Tierney y Cunningham (1984) definen la analogía como "un método expositivo para comparar núcleos de información que son sirnilares en aspectos esenciales y que permiten trasladar atributos desde la información familiar a la desconocida".

Se postula que en Ciencias Naturales la utilización de analogías es útil para ayudar al alumno a construir esquemas en topicos desconocidos (Tierney y Cunningham 1984, Bean, Singer y Cowan 1985).

Otros estudios realizados en este campo han demostrado que si bien las analogías en un texto de ciencias ayudan a la comprensión de los conceptos, también es necesario que el profesor enseñe el mecanismo mental que el estudiante debe seguir para transferirlos ( $\mathrm{Si}$ mons 1984).

Bean y col. (1985) señaian que ei profesor debe trabajar tres aspectos antes de usar la analogía como auxiliar de la comprensión de textos en ciencias: (1) analizar el texto que apoyará la enseñanza de los conceptos claves, (2) crear analogías apropiadas y elaborar con ellas una guía de estudio, y (3) dar instrucciones a los estudiantes acerca de la forma de usar la guía y el texto.

Investigaciones realizadas en relación a la eficacia de utilizar textos que refuten los pre-conceptos como un medio para eliminarlos y sustituirlos por los conceptos científicos adecuados han permitido comprobar efectos significativos, sobre todo cuando se enfrenta al estudiante con redes conceptuales complejas. (Hund y Alvermann 1986). En esta línea de trabajo se han propuesto dos estrategias para ayudar a los estudiantes a abandonar sus concepciones espontáneas o pre-conceptos utilizando textos.

Una de ellas propone utilizar una guía de reacción anticipada. Ésta consiste en un conjunto de afirmaciones relacionadas con el tema científico que se estudiará: (I) frente a cada afirmación el alumno deberá responder si o no utilizando sólo su conocimiento previo, (2) en segundo término, se procede a discutir las respuestas dadas por los estudiantes, (3) a continuación se les pide que lean el texto preparado por el profesor indicándoles que vayan destacando las posibles discrepancias que encuentren con respecto a sus respuestas anteriores, (4) una vez hecho este ejercicio se les invita a releer sus respuestas espontáneas para descubrir y si es necesario discutir con el educador las diferencias. (Readence, Bean, y Baldwin 1981).

La estrategia propone trabajar en cuatro etapas que se inician (1) con una discusion a partir de una pre-lectura de un texto preparado para producir desequilibrio en el alumno. Al descubrir que sus pre-conceptos no pueden dar explicación al hecho planteado, se le invita a (2) leer un segundo texto que le permita tomar contacto con el concepto adecuado. Éste debe explicar los hechos planteados en el primer documento. Una vez realizada la lectura deben demostrar que ellos la comprenden a través de la ejemplificación adecuada del fenómeno o concepto científico central presentado. El educador debe (3) ayudar a los estudiantes a comparar sus pre-conceptos con los conceptos científicos, para descubrir las deficiencias de los primeros (Posner, Strike, Hewson y Gertzog 1982).

Se ha demostrado también que la utilización de estrategias metacognitivas, al enfrentar al estudiante con un texto cientifico que presenta nuevos conceptos, tiene muy buenos resultados porque mejora la comprensión.

Para fomentar este proceso se sugiere que (I) después de una sesión inicial de orientación destinada a la activación de los esquemas cognitivos de los alumnos, se debe destinar algo de tiempo para que (2) en pequeños grupos revisen sus ideas en torno al tema que se está tratando, explícitándolas a través de un póster o utilizando otros medios.

(3) Posteriormente se exponen sus resultados y se realiza un paralelo destacando los aspectos que necesi. tan una posterior consideración. (4) En ese momento pueden enfrentarse al texto para comparar sus ideas con las que en éste se exponen. Se recomienda también que escriban anotaciones personales sobre lo que ha ocurrido durante el proceso de aprendizaje (Driver 1988).

En la misma línea de las estrategias metacognitivas como vías para mejorar la comprensión y recuerdo de la información en ciencias se circunscribe el planteamiento de Roger Anderson (1986). De acuerdo con Anderson, para que una comunicación en ciencias, oral o escrita, sea fácil de procesar y registrar mentalmente debe poseer un fluido equilibrado entre conceptos científicos que son conocidos y se repiten en la comunica* ción y los que se van incorporando. Desde esta perspectiva la estructura de una comunicación científica debería contemplar oraciones contiguas que contienen material verbal común. Así la adquisición de un contenido facilitará proactivamente la adquisición del siguiente. Como la comunicación verbal es serial, la presencia de conceptos científicos en unidades de dis- 
curso contiguas se denomina comunalidad. Cuando se presentan elementos verbales nuevos en el texto se produce una progresión. Si un texto contiene excesiva progresión y reducida comunalidad pierde eficiencia en términos de medio de aprendizaje científico. Es necesario un grado de progresion acorde con la capacidad de aprendizaje del estudiante, si ésta es muy rápiđa impide la comprensión y si es muy lenta, produce disminución de la atención (Sánchez 1987).

El método de experiencias de lenguaje es también otra forma de crear puentes entre las actividades realizadas por los niños en las clases de Ciencias Naturales y la comprensión de los conceptos científicos. Éste consiste en estimular a los alumnos a preguntar y responder usando indistintamente las cuatro manifestaciones del lenguaje: hablar, escuchar, escribir y leer. (Borrow, Kristo y Andrew 1984).

Acorde con el enfoque constructivista de la enseñanza de las Ciencias Naturales, la utilización de la exposicion oral y escrita de los contenidos es inapropiada para el niño en etapa de desarrollo pre-operacional y operacional concreto. Porque el lenguaje puede transmitir conceptos y relaciones que no están aún a nivel de la comprensión del alumno (Duckworth 1964).

Como una forma de mejorar esta dificultad, Ester y Merritt (1976) plantean que es factible utilizar las necesarias experiencias de los niños con actividades

\section{REFERENCIAS BIBLIOGRÁFICAS}

ANDERSON R. y PEARSON P.D. A Schema theoretic: View of Basic Processes in Reading Comprehension, en Handbook of reading research. (D. Pearson: N.Y. Longman).

ANDERSON, R., 1984. Role of the reader's schema in comprehension, learning and memory, en Learning to read in american schools: Basal readers and content texts. ( $\mathrm{Ri}-$ chard C. Anderson: N.Y. Lawrence Eriboum).

AUSUBEL, D.P., 1986. Educational Psychology: a cognitive view. (Holt, Rinehart and Winston: New York).

BARROW Ll. y ANDREW, B., 1981. Activity Oriented Science plus language experience approach. 29 Annual Meeting of the National Scie-ce Teacher's Association. New York.

BARROW, Ll., KRISTO, J. y ANDREW, B., 1984. BuIIding bridges between Science and Reading, The Reading Teacher, pp. 179-193.

BEAN, T., SINGER, H. y COWAN, S., 1985. Analogical study guides: Improving comprehension in Sciences, Journal of Reading. Vol. 29(3), pp. 246-250. manipulativas en ciencias como una valiosa forma de comunicar sus observaciones a través de experiencias de lenguaje. Estas actividades motivan al niño a leer en función de la necesidad de responder a sus observaciones, incentivan entonces el deseo de leer textos de ciencias y aproximan al niño a la comprensión de conceptos científicos y sus relaciones.

Borrow y Andrew (1981) proponen una estrategia de "Experiencia de Lenguaje en Ciencia" (S.L.E.) que consta de 5 etapas: (1) El profesor provee material para que el alumno manipule y utilice al mismo tiempo preguntas que lo llevan al plano del análisis y de la discusión. (2) Los niños comparten sus observaciones y las escriben. (3) Interpretan sus observaciones a través del dibujo. (4) Comparten sus ilustraciones y se comunican sus descubrimientos. (5) Buscan información adicional para corroborar o rechazar sus hipótesis o sus interpretaciones. Esta estrategia, además de permitir un trabajo científico por descubrimiento a partir de los esquemas conceptuales del niño, permite aumentar el conocimiento del vocabulario y mejora la comprension. Esta forma de enseñar ciencias en educación básica demuestra al estudiante que la ciencia, además de entretener, le brinda información. La experiencia de lenguaje en ciencias permite desarrollar también, habilidades propias de las disciplinas científicas tales como observar, clasificar, medir, inferir, predecir, interpretar datos y comunicar.
BRITTON, B., GLYNN, S., MEYER, B. y PENLAND, M. 1982. Effects of text siructure on use of cognitive capacity during reading, Journal of Educational Psychology, 74, pp. 51-61.

CARRETERO, M., 1987. A la búsqueda de la genesis del método cientiffico: un estudio sobre la capacidad de eliminar hipotesis, Infoncia y Aprendizaje, 38, pp. 53.

CARRETERO, M. y GARCIA MADRUGA, J., 1987. Lecturas de psicologia del pensamiento, Infancia y aprendizaje, 38, pp. 46. (Editorial Alianza: Madrid, 1984).

COSGRAVE, M. y OSBORNE, R., 1985. Lesson Frameworks for changing children's ideas, en R. Osborne y P. Freyberg, Learning in science. (Heinemann: Hong-Kong).

CASSELS, J., JHONSTONE, H., 1983. The meaning of words and the teaching of Chemistry, Education in Chemistry, Jan. 10.

CASTAÑEDA, S., LÓPEZ, M. y ROMERO, M. The role of five induced learning strategies in scientific text comprehension, The Journal of Experimental Education, Vol. $55(3)$, pp. $125-130$. 
CASTAÑEDA, S., LÓPEZ, M. y ROMERO, M., 1987. The role of five induced learning strategies in scientific text comprehension, The Journal of Experimental Education, Vol. 55(3), pp. 125-130, spring.

DRIVER, R., 1986. Psicología cognoscitiva y esquemas conceptuales de los alumnos, Enseñanza de las Ciencias, $4(1)$, p. 3.

DRIVER, R., 1988. Un enfoque constructivista para el desarrollo del currículo en ciencias, Enseñanza de las Ciencias, Vol. 6(2), pp. 109-120.

DUCKWORTH, E., 1964. Piaget Rediscovered, Journal of Research in Science Teaching, Vol. 2, pp. 172-175.

ESTER, W. y MERRIT, K., 1976. Teaching reading through Science Experience Studies, School Science and Mathematics, Vol. 76, pp. 203-206.

GAGNÉ, R. y BRIGGS, L.J., 1978. La planificación de la enseñanza. Sus principios. (Editorial Tritlas: México).

GAMBLE, R., 1986. Simple equations in phisics, European Journal Science Education, (1), pp. 27-37.

GARDNER, P.L., 1972. Words in Science, Australian Science Education, Proyect. Melbourne.

GILBERT, J., OSBORNE, R., 1980.1 understand, but I don't get it. Some problems of learning science, School Science Review, 61(218), pp. 664-674.

HAWKINS, D., 1978. Critical Barriers in Science Learning, Outlook, 29, pp. 3-22.

HERRON, J.D., 1979. Hey, eatch your language, Journal of Chemical education, Vol. 56 , pp.330.

HOLLEY, C.D., DONSEREAU, D.F. y FENDER, R.M., 1981. Some data and comments regarding educational set theory, Journal of Educational Psychology, 73, pp. 494504.

HYND, C. y ALVERMANN, D., 1986. The role of refutation text in overcoming difficulty with science concepts, Journal of Reading, vol. 29(8), pp. 440-447.

LAWSON, A.E.; SEPHERD, G.D., 1979. Syntactical Complexity in Written Language and Cognitive Development at Formal Level, Science Educational, 63(1), pp. 73-81.

LLORENS, J., LLOPIS, R. y DE JAIME Ma. C., 2987. El uso de la terminología científica en Ios alumnos que comienzan el estudio de la química en la enseñanza media. Una propuesta metodológica para su análisis, Enseñanza de las Ciencias, Vol. 5(1), pp. 33-40.

LURIA, A.R., 1980. Lenguaje y Pensamiento. (Editorial Fontanella: Barcelona).

MEDWAY, P., 1987. Understanding Children Talking. (Penguin: London), 1976. Citado en Enseñanza de las Ciencias, Vol. 5(1), p. 33.
MOREIRA, M. y NOVAK J., 1988. Investigación en enseñanza de las ciencias en la Universidad de Conell: Esquemas teóricos, cuestiones centrales y abordes metodológicos, Enseñanza de las Ciencias, 6(1), pp. 3.18.

MORENO, F.P., 1985. Enseñanza y aprendizaje de conceptos en la adolescencia, Estudios Pedagógicos, 11(27).

NUSSBAUM, J. y NOVICK, S., 1971. Brainstorning in the classroom to invent a model: a case study, School Science Review, 72, p. 771.

OSBORNE, R, y FREYBERG, P, 1987. Learning in science. (Heinemann: Hong-Kong), 1985. Citado en Infancia $y$ Aprendizaje, 38, 43 .

OTERO, J. y BRINCONES, I., 1987. El aprendizaje significativo de la segunda ley de la termodinámica, Infancia y Aprendizaje, 38.

POSNER, G., STRIKE, K., HEWSON, P, y GERTZAG, W., 1982. Accommodation of a scientific conception: Toward a theory of conceptual change, Science Education, Vol. 66 , pp. $211 \cdot 217$

POZO, J., 1987. Y, sin embargo, se puede enseñar ciencia, Infancio y Aprendizaje, 38, p. 109.

POZO, J.L., 1987. Aprendizaje de la ciencia y pensamiento causal. (Editorial Visor: Madrid).

POZO, J. y CARRETERO, M., 1987. Del pensamiento formal a las concepciones espontáneas: $¿$ qué cambia en la enseñanza de la ciencia?, Infancia y Aprendizaje, 38, p. 35.

READENCE, J., BEAN, T. y BALDWIN, S., 1981. Content area reading: an integrated approach. (Dubugue, Yowa: Kendall/Funt Publishing Co).

SANTESTEBAN, A.J. y KORAN, J.J., 1977. Instruccional adjuncts and learning science from written materials, Journal of Research in Science Teaching, 1, pp. 51-55.

SAPIR, E., 1970. Le language. (Payot: París).

SIMONS, P., 1984. Instructing with analogies, Journal of Educational psychology, Vol, 76, pp. 513-527.

STUART, J., 1982. An identification of life science concepts in selected secondary school science texbooks, School Science and Mathematics, 82, pp. 189-200.

TIERNEY, R. y CUNNINGHAM, J., 1984. Research on teaching reading comprehension, en Pearson, D. (ed.), Handbook of Reading Research._(Longman: New York). p. 613.

VAN DIJK, T. y KINTSCH, W., 1983. Strategies of discourse comprehension. (Academic Press: New York).

VYGOTSKI, L.S., 1962. Thought and Language. (Mit Press: Cambridge).

WEIDLER, S., 1984. Reading in the content area of science. (Mary M. Duptuis editor: I.R.A.), pp. 54-65.

WHORF, B., 1971. Language. Thougt and Reality. (Mit Press: Cambridge). 